\title{
On The Time Scale of Internal Energy Relaxation of AP-MALDI and nano-ESI Ions in a Quadrupole Ion Trap
}

\author{
Philip M. Remes and Gary L. Glish \\ Department of Chemistry, University of North Carolina at Chapel Hill, Chapel Hill, North Carolina, USA
}

Recently reported results (Konn et al. [14]) on the collisional cooling of atmospheric pressure matrix assisted laser desorption ionization (AP-MALDI) and nano-electrospray ionization (nano-ESI) generated ions in a quadrupole ion trap mass spectrometer (QITMS) are inconsistent with measured collisional cooling rates. The work reported here presents a re-examination of those previous results. Collision induced dissociation (CID) has been used to probe various properties of ions contained in a QITMS. It is shown experimentally that when trapping large numbers of ions, an effective dc trapping voltage is induced that varies with changes in the size of the ion cloud. A decrease in the resonant frequency for maximum CID efficiency is observed as the cool time between parent ion isolation and CID is increased. Ion trajectories in a QITMS are simulated to demonstrate how ion density changes over the course of parent ion isolation. The effect of space charge on ion motion is simulated, and Fourier transformations of ion axial motion plus simple calculations corroborate the experimentally observed transient frequency shifts. The relative stability of ions formed by AP-MALDI and nano-ESI is compared under low charge density conditions. These data show that the ions have reached equilibrium internal energy and, thus, that differences in dissociation onsets and " $50 \%$ fragmentation efficiency points" between the ionization mechanisms are due to the formation of distinct ion conformations as previously shown in reference [28]. The conclusions of Konn et al. [14] are based on invalid experimental procedures as well as inappropriate comparisons of QITMS data to low-pressure FT-ICR data. (J Am Soc Mass Spectrom 2009, 20, 1801-1812) (@ 2009 American Society for Mass Spectrometry

$\mathrm{A}$ $\mathrm{n}$ understanding of ion internal energy is of primary interest to researchers studying the structures of gas-phase ions. How fast an ion will dissociate, which mechanisms will be involved, and how many products will be observed all have a large dependence on the amount of internal energy in the ion and the experimental time window for dissociation [1-4]. If the internal energy of an ion is distributed statistically among its bonds, RRKM theory can determine the dissociation kinetics of the various dissociation pathways, and mass spectra or tandem mass spectra (MS/MS) could be predicted [5-7]. MS/MS spectra are related to ion structure on a fundamental level because the vibrational bond frequencies, the ground state energy, and the critical energy of dissociation are all determined on some level by the three dimensional conformation of the ion.

Because MS/MS spectra have a dependence on structure and internal energy, it is important to understand the various factors that determine ion internal energy and how its magnitude might change over the course of an experiment. A typical experiment using a

Address reprint requests to Professor G. L. Glish, University of North Carolina at Chapel Hill, CB no. 3290 Caudill Laboratory, Chapel Hill, NC 27599, USA. E-mail: glish@unc.edu quadrupole ion trap mass spectrometer (QITMS) lasts several hundred ms, during which time a large number of variables can affect an ion population's distribution of internal energy. The theoretical calculation of ion internal energy in a QITMS is a complex quantum chemical calculation in which many factors must be considered, such as the kinetic energy of the ion, the vibrational energy states of the ion, and the probability of energy-transfer from one state to another [8]. These considerations are complicated by the necessary presence of helium (He) bath gas at $\sim 1.3 \times 10^{-3}$ mbar in the QITMS to improve sensitivity and mass resolution [9]. Other types of mass spectrometers typically operate at pressures low enough to ensure that the flight path of the ion is shorter than the mean free path between collisions with background neutral gas. Conversely, in a QITMS, with several tens of collisions per ms, an ion may experience many thousands of collisions over its lifetime in the instrument [10]. Each of these collisions has the potential for changing the internal energy of the ion, ultimately having an effect on the mass spectrum that will be produced.

Indeed, the collision of an ion with a neutral gas molecule is one of the most important means for energy-transfer in mass spectrometry, with the most common application of this process being collision 
induced dissociation (CID). When an ion collides with a neutral molecule such as He, kinetic and internal energy may be inter-converted. Because energy is conserved, eq 1 can be written, where $\mathrm{KE}_{0}, \mathrm{KE}_{\mathrm{F}}, \mathrm{IE}_{0}$, and $\mathrm{IE}_{\mathrm{F}}$ are, respectively, initial kinetic energy, final kinetic energy, initial internal energy, and final internal energy of the ion and colliding $\mathrm{He}$ atom. He, of course, being monoatomic, has no internal energy under typical QITMS conditions.

$$
K E_{0}^{I O N}+I E_{0}^{I O N}+K E_{0}^{H E}=K E_{F}^{I O N}+I E_{F}^{I O N}+K E_{F}^{H E}
$$

The change in internal energy of the ion is the quantity of interest, and so eq 1 may be rearranged to eq 2 , where $\Delta$ denotes the final value minus the initial value. Consideration of eq 2 shows that an ion may be excited in a collision through a conversion (reduction) of either the ion's or He's kinetic energy, and conversely an ion may be cooled by transferring its internal energy into an increase in kinetic energy of the ion or He.

$$
\Delta I E^{I O N}=-\Delta K E^{I O N}+-\Delta K E^{H E}
$$

Whether an ion gains or loses internal energy in a collision depends on the probability of energy-transfer between states, and is a problem that has been considered theoretically for a QITMS [8, 11]. In particular, the evolution of peptide internal energy with time due to collisional cooling in a QITMS has been investigated [11]. Plots of calculated internal energy versus time revealed that a series of homologous peptide ions $(\mathrm{AG})_{\mathrm{n}}$, where $n=8-32$ ) excited to $450 \mathrm{~K}$ would all relax to room temperature in less than $10 \mathrm{~ms}$. Experiments designed to test this theoretical treatment have been published recently, where two laser pulses are used to probe ion internal energy as a function of time [12, 13]. Ions were irradiated with one laser pulse and allowed to cool for a set amount of time before being irradiated again. During the time between pulses, the ions lose internal energy to collisions with $\mathrm{He}$, causing the second laser pulse to dissociate a smaller percentage of parent ions. Using the efficiency of fragmentation as a measure of internal energy, relaxation times for excited ions at typical operating pressures of 9.3-1.5 $\times 10^{-3}$ mbar were observed to be about 5 to $20 \mathrm{~ms}$.

Recently, a study has been reported that concludes internal energy relaxation of peptide ions through $\mathrm{He}$ collisions to be a relatively inefficient process requiring several hundred $\mathrm{ms}$ when the experiment is conducted in a QITMS [14]. Supporting the results was a cited observation that the process of internal vibrational relaxation through collisions is $\sim 100$ times slower than collisional kinetic energy relaxation. If true, the conclusion could be drawn that if kinetic relaxation takes place in 1-10 ms, then internal energy relaxation must take place in 100-1000 ms. The referenced study described the vibrational relaxation of $\mathrm{N}_{2}{ }^{+\cdot}$ through collisions with a variety of noble gases, in which the cooling process with He was least efficient [15]. However, it would be an over-simplification to assume that the collision induced vibrational relaxation behavior of $\mathrm{N}_{2}{ }^{+}$, which has one vibrational degree of freedom and a vibrational density of states of 1 per $\mathrm{cm}^{-1}$ at room temperature, could be used as an accurate predictor of the collision induced vibrational relaxation behavior of a medium sized peptide such as leucine enkephalin (YGGFL), which has 228 degrees of freedom and a vibrational density of states of $3.4 \times 10^{36}$ per $\mathrm{cm}^{-1}$ at room temperature, calculated with the direct count method [16] As our group has an interest in the mechanisms and kinetics of internal energy-transfer processes in mass spectrometry experiments, the study described here undertakes a re-examination of the cited work.

An important experimental parameter that could be responsible for the very long predicted relaxation times described in the previous study could be the use of long ion accumulation, or gate, periods $(400 \mathrm{~ms})$, and it is on this point that the current investigation will focus. The long ion accumulation time was justified by a desire to accumulate equal numbers of ions over equal lengths of time in a comparison of the internal energies of ions produced by atmospheric pressure matrix assisted laser desorption ionization (AP-MALDI) and nano-electrospray ionization (nano-ESI). Because a lower flux of ions was produced by AP-MALDI, and the QITMS has a finite trapping capacity, it was reasoned that the trapping volume could be "overfilled" in the high ion flux, nano-ESI case such that after $400 \mathrm{~ms}$, equal numbers of ions would be accumulated using both ionization methods. Undeniably, a plot of ion abundance versus ion accumulation time will show abundance leveling off after a time, so the authors' supposition of equal ion populations for both ionization techniques could be accurate. However, operation of a QITMS under high space charge conditions introduces a new set of variables with respect to ion motion, namely, an added dc potential. A brief analysis of the stability equations for a QITMS will demonstrate the effect of this new dc potential. The canonical Mathieu formulae for the so-called axial stability parameters in the QITMS, $\mathrm{a}_{\mathrm{z}}$ and $\mathrm{q}_{\mathrm{z}}$, are given in eqs 3 and 4 , where e is the fundamental unit of charge, $\mathrm{V}$ and $\mathrm{U}$ are the amplitudes of the maximum zero-to-peak radio frequency (rf) and dc trapping potentials, $m$ is mass, $r_{0}$ is the radius of the ring electrode, $z_{0}$ is axial spacing of endcap electrodes, and $\Omega$ is the angular frequency of the rf trapping voltage [17].

$$
\begin{aligned}
& q_{z}=\frac{8 e V}{m\left(r_{0}^{2}+2 z_{0}^{2}\right) \Omega^{2}} \\
& a_{z}=-\frac{16 e U}{m\left(r_{0}^{2}+2 z_{0}^{2}\right) \Omega^{2}}
\end{aligned}
$$

The fundamental frequency of ion oscillation, or secular frequency, is given by eq 5 , where $\beta_{z}$ is given in eq 6 when $\mathrm{q}_{\mathrm{z}} \leq 0.4$. 


$$
\begin{aligned}
& \omega_{z}=\frac{1}{2} \beta_{z} \Omega \\
& \beta_{z}=\left(a_{z}+\frac{1}{2} q_{z}^{2}\right)^{\frac{1}{2}}
\end{aligned}
$$

Using eqs 4-6, one observes that a positive $U$ will decrease an ion's secular frequency. Traditionally, U would be induced instrumentally, but a dc potential can also be induced by a collection of charged particles of the same polarity. However, a potential induced by a cloud of charged particles is expected to vary as the density of the cloud changes. The ion isolation event immediately preceding CID provides an opportunity for a change in ion cloud density. During isolation, the ion cloud will experience several different trapping environments, most notably a gain in kinetic energy as the $q_{z}$ value approaches a resonant ejection point, and a decrease in the depth of the pseudopotential trapping well as $q_{z}$ is reduced to the value used for CID. The depth of the pseudopotential well is given in eq $7[17,18]$.

$$
D_{z}=\frac{m q_{z}^{2} \Omega^{2} z_{0}^{2}}{16 e}
$$

The purpose of the present investigation is to demonstrate experimentally and theoretically that these varying trapping environments lead to changes in ion secular frequency when a large population of ions is present. When CID is performed using a single resonant excitation frequency on a population of ions whose secular frequency changes, internal energy measurements based on fragmentation efficiency taken at different times are inevitably inaccurate.

\section{Methods}

\section{AP-MALDI and nano-ESI on Bruker Esquire}

Experiments were carried out on two instruments, a Bruker (Billerica, MA) Esquire ion trap using OS2 operating software for AP-MALDI and nano-ESI experiments, and also a modified Finnigan (San Jose, CA) ITMS that has been described previously [19], used for additional experiments with nano-ESI. The Bruker Esquire has a modified Analytica (Branford, CT) electrospray ionization source, which has been fitted with a flared glass capillary [20, 21]. The flared capillary coupled with a Lexan sample holder and Plexiglass mounting assembly create a simple aerodynamic AP-MALDI source [22, 23]. Matrix/analyte solutions are introduced into tapered through-holes in the Lexan sample holder. After drying, crystals remain deposited around the edges of the holes, whereupon the sample holder is placed flush with the open flared capillary and sealed with a rubber o-ring around the capillary. $\mathrm{A} \mathrm{N}_{2}$ laser (VSL-337-NDS; Laser Science Inc., Franklin, MA) is focused into fiber optics for delivery of $337 \mathrm{~nm}$ light to the sample with measured energy of $\sim 20 \mu \mathrm{J}$ per pulse (RM-6600 Universal Radiometer; Laser Precision Corp., Irvine, CA). The laser was triggered with a pulse generator at $10 \mathrm{~Hz}$ (8013B \#; Hewlett-Packard, Santa Clara, CA) that in turn is triggered by a signal generated by the Bruker hardware at the start of the ion accumulation portion of the experiment. The same flared capillary is used for nano-ESI by placing a metal cap with a $0.5 \mathrm{~mm}$ hole over the capillary, to which is applied a voltage of $-1000 \mathrm{~V}$ for production of positive ions. Sample solutions are introduced into a pulled glass needle that contains a grounded wire introduced through the back end of the needle, and the tip of the needle is placed $\sim 1-3 \mathrm{~mm}$ from the opening of the metal cap. The relative stability of AP-MALDI and nano-ESI generated ions was compared by dissociating the ions via CID (with an incremented excitation waveform amplitude) and recording fragmentation efficiency as shown in eq 8 , where $\Sigma F$ is the sum of fragment ion abundance and $\Sigma \mathrm{P}$ is the "survivor" parent ion abundance.

$$
F E=\frac{\Sigma F}{\Sigma F+\Sigma P}
$$

Figure 1 illustrates the scan function (rf trapping voltage applied to the ring electrode changes versus time) for the ITMS experiments. The rf trapping voltage is proportional to the lowest $m / z$ trapped, or "start mass" at any particular time. This relationship allows "start mass" or "low mass cut-off" to be used interchangeably with "rf voltage" as the y-axis label. These former terms are more commonly used than the latter when referring to various aspects of a scan function. Details about the Bruker scan function were obtained by using the disconnected high-voltage dynode as a capacitive "pickup." The resonance ejection voltage was removed from its feedthrough and the endcap electrodes grounded, so that a digital oscilloscope could be used to monitor the rf

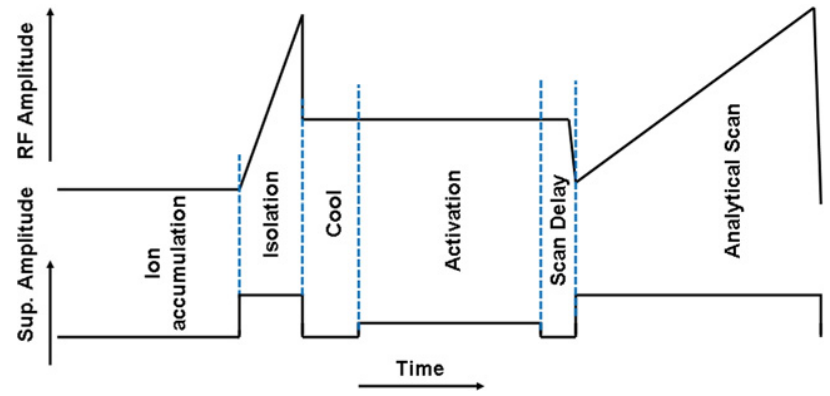

Figure 1. Scan function for Finnigan ITMS showing rf trapping voltage as a function of time, and the timing of supplementary waveforms for ion ejection and excitation. Typical times are as follows, gate (ion accumulation) $=30 \mathrm{~ms}$, isolation $=10 \mathrm{~ms}$, cool time $=30 \mathrm{~ms}$, activation $=40 \mathrm{~ms}$, scan delay $=5 \mathrm{~ms}$. All segments are drawn to scale except the analytical scan segment, which is 13 $\mathrm{kDa} / \mathrm{s}$ for the Bruker Esquire and $5555 \mathrm{Da} / \mathrm{s}$ for the Finnigan ITMS. 
voltage on the high-voltage dynode. The Bruker scan function takes about $90 \mathrm{~ms}$ to isolate the ions, with the remainder of the scan function being very similar to that of the ITMS. A "start mass" of 80 Da was used for all ions studied during the accumulation time on the Bruker. Ions were isolated with a $\pm 10 \mathrm{Da}$ window, and CID was conducted with a $\mathrm{q}_{\mathrm{z}}$ value of 0.25 . CID activation was performed either with the basic scan function depicted in Figure 1 or, alternatively, with no ion isolation, and FE could be plotted, where $\Sigma$ F in eq 1 is replaced by the intensity of just one selected product ion. To gauge the effect of cooling time on ion dissociation, the time before CID in the scan function was incremented manually for each acquired spectrum. Spectra were acquired as the average of 20 scans, and error bars were calculated as $95 \%$ confidence intervals on 5-8 experiment repetitions the same day. To minimize the effects of space charge while standardizing conditions as much as possible between AP-MALDI and nano-ESI, the same ion accumulation time, or "gate time" of $30 \mathrm{~ms}$ was used in both cases. Because many fewer ions are produced by AP-MALDI than by nanoESI over a given time period for the current experiments, the rf voltage amplitude of the transfer hexapole was decreased for nano-ESI to equalize the ion populations between the two experiments. In most cases, the maximum $700 \mathrm{~V}_{\mathrm{p}-\mathrm{p}}$ hexapole voltage was used for AP-MALDI, and $\sim 100 \mathrm{~V}_{\mathrm{p} \text {-p }}$ was used for nano-ESI. A 30 $\mathrm{ms}$ ion accumulation time only allows time for one laser pulse for AP-MALDI, but this was enough to accumulate a sufficiently large ion population for these experiments. The ion abundance of the parent ion for the Bruker experiments was $\sim 3-5 \times 10^{3}$ (in terms of the Bruker software).

A direct reading of the pressure inside the ion trapping volume is not possible for the Bruker Esquire because transport of the He bath gas to the ionization pressure gauge is attenuated by conductance limiting electrode spacers. However, previous experiments using the ion/molecule reaction of cytochrome $c$ with dimethylamine have been performed to calibrate the pressure gauge [22]. These experiments showed a factor of $\sim 100 \times$ between ionization gauge pressure and ion trapping volume pressure for the Bruker Esquire. The present experiments were performed at a pressure reading of $3.0 \times 10^{-5}$ mbar, with the pressure in the trap estimated to be about $3 \times 10^{-3}$ mbar.

\section{Sample Preparation}

The peptides desArg ${ }^{1}$ bradykinin (PPGFSPFR), desArg ${ }^{9}$ Bradykinin (RPPGFSPF), Sar-Angiotensin II (SarRVYIHPF), and angiotensin III antipeptide (GVYVHPV) were purchased from Sigma Aldrich (St. Louis, MO) and used without purification. Solutions of 79/20/1 vol/ $\mathrm{vol} / \mathrm{vol}$ methanol/water/acetic acid were made at 100$200 \mu \mathrm{M}$ for nano-ESI. The same solutions were combined in a 1:4 ratio with $100 \mathrm{mM}$ 2,5-dihydroxybenzoic acid (DHB) in 1:2 $\mathrm{H}_{2} \mathrm{O}, \mathrm{CH}_{3} \mathrm{CN}$, or $100 \mathrm{mM} \alpha$-cyano-4- hydroxycinnamic acid (CHCA) in 1:2.3 $\mathrm{H}_{2} \mathrm{O}, \mathrm{CH}_{3} \mathrm{CN}$, giving a 1:4000 analyte to matrix ratio. The analyte/ matrix solution was deposited in $15 \mu \mathrm{L}$ aliquots in each of the tapered-holes in the Lexan sample plate and allowed to dry.

\section{Higher Time Resolution Cooling Study}

The Finnigan ITMS was used to examine the effect of a cool time on CID for nano-ESI ions. The ITMS software controls all scan function parameters with microsecond resolution. Cooling time and CID resonant excitation frequency were varied automatically under software control. A "start mass" between 40 and 60 Da was used in the ITMS experiments. Isolation was achieved by increasing the rf amplitude for $10 \mathrm{~ms}$ to eject ions having $m / z$ less than the parent ion; no attempt was made to eject ions with higher $m / z$ as these were of an insignificant overall abundance. CID was conducted at a $\mathrm{q}_{\mathrm{z}}$ value of 0.253 , and at a resonant excitation frequency corresponding to the maximum achievable fragmentation efficiency. A plot of fragmentation efficiency versus resonance excitation frequency is sometimes referred to as a resonance absorption curve. Such curves are important to assure any shifts in resonant frequency due to space charge or amplitude of the resonant excitation voltage. Spectra were acquired as the average of 25 scans, and error bars were calculated as $95 \%$ confidence intervals on 5 experiment repetitions the same day. The electrodes do not have conductance limits to gas transport, so the measured ionization gauge pressure is the ion trapping volume pressure, after correction for the ionization potential of He (i.e., multiplying by 7 to correct for the lower gauge sensitivity). A pressure of $9.3 \times 10^{-4} \mathrm{mbar}$ of He was used for the experiments.

\section{Ion Trajectory Calculations}

Ion motion inside the QITMS was simulated using SIMION 7.0 [24] using a Pentium 4, 3.06 GHz personal computer. The values for radius of the trapping volume and the axial dimension were 1.000 and $0.783 \mathrm{~cm}$, respectively, which correspond to Finnigan ITMS dimensions. Simulation quality was set to 3 for all simulations, and grid spacing was $0.05 \mathrm{~mm}$. Ions were placed in the center of the trapping volume $\pm 1.0 \mathrm{~mm}$ at a random phase of the $1.1 \mathrm{MHz}$ trapping potential, and with initial kinetic energy of $0.15 \mathrm{eV} \pm 5 \%$ in a random direction. Collisions with $9.3 \times 10^{-4}$ mbar He neutral gas were simulated using the $3 \mathrm{D}$ hard sphere model developed by Manura [25]. Interactions between ions could be simulated with the SIMION point charge model. In this mode, a total amount of charge is designated to be distributed amongst all the ions being simulated. For example, if 10 ions were being simulated and the total charge was $1.6 \times 10^{-16} \mathrm{C}$, then each affects the others as if it has the charge of 100 ions, and it 
would be expected that the ions behave as if there were 1000 total ions in the trap. The $\mathrm{x}, \mathrm{y}, \mathrm{z}$ positions and kinetic energy of the ions were recorded every $0.1 \mu$ s for analysis of ion motion, which was carried out in software written in LabView 7.1 (National Instruments Corp., Austin, TX).

\section{Theory}

\section{Calculation of Space Charge Effects}

Some of the effects of ion interactions can be estimated using calculations having various degrees of sophistication. Most pertinent to the present experiments is an estimation of the dc voltage magnitude induced by a group of ions, which can result in a shift in ion secular frequency. The voltage induced at a particular distance (radius) $r$ away from a point charge $q$ is given by the first term in eq 9, where $\varepsilon_{\mathrm{o}}$ is the permittivity of vacuum. The voltage induced by a number of point charges can be expressed as a sum as in eq 9 .

$$
V=\frac{q_{1}}{4 \pi \varepsilon_{0} r_{1}}+\frac{q_{2}}{4 \pi \varepsilon_{0} r_{2}}+\ldots
$$

\section{Sphere of Constant Ion Density}

The simplest case calculation assumes that the ions are distributed uniformly in a sphere of a given radius. In a general sense, the voltage induced by a volume charge is given in eq 10,

$$
V=\frac{1}{4 \pi \varepsilon_{o}} \int \frac{\rho}{r} d \tau
$$

where $\rho$ is charge density, $\mathrm{r}$ is distance to a particular point, and $d \tau$ is the volume element [26]. The integral can be numerically approximated as shown in eq 11 , where $\mathrm{N}$ is number of ions, $\mathrm{r}_{\mathrm{s}}$ is the radius of the sphere, and $r_{i}$ is the radius of each particular volume element.

$$
V=\frac{1}{4 \pi \varepsilon_{0}} \sum_{i} \frac{N q}{r_{s}^{3}} \frac{1}{r_{i}} \cdot\left[r_{i}^{3}-r_{i-1}^{3}\right]
$$

\section{Gaussian Distribution}

A more realistically shaped ion cloud can be described using a Gaussian distribution [27-29]. In this case, higher dc voltages would be expected in the center of a Gaussian distribution of charges than in the center of a sphere of constant charge density of similar size because in the former case most of the ions are located at the center of the distribution. Given a distribution of a given size and a certain number of ions, the magnitude of induced voltage at the center can be estimated using eq 12 , where $p$ is the width of each slice of the distribution, and $\sigma$ is the standard deviation of the distribution.

$$
\begin{aligned}
V= & \frac{2}{4 \pi \varepsilon_{0}} \cdot \sum_{n=0}^{\infty} \sum_{r=n p}^{r=(n+1) p} \frac{1}{\sigma \sqrt{2 \pi}} \\
& \times \exp \left[-\frac{r^{2}}{2 \sigma^{2}}\right] \cdot \frac{N q}{\left(\frac{n p+(n+1) p}{2}\right)}
\end{aligned}
$$

\section{Results and Discussion}

\section{Comparison of AP-MALDI Versus nano-ESI}

Figure 2 shows the results of experiments done using the Bruker Esquire. The breakdown curves of fragmentation efficiency versus CID excitation voltage show that for the peptides studied, the onset of dissociation and $50 \%$ fragmentation efficiency point for AP-MALDI comes at an equal or greater energy than for nano-ESI. One conclusion based on these data is that the APMALDI ions have either slightly lower internal energies or slightly higher critical energies of dissociation. A previous study of the dissociation onset energies of liquid secondary ion mass spectrometry (LSIMS) ions versus nano-ESI ions found that the nano-ESI ions dissociated at lower energies than the LSIMS ions, suggesting that the LSIMS ions had either lower internal energies, or higher critical energies of dissociation [30]. However, in the LSIMS case, as well as the present AP-MALDI case, the hypothesis that nano-ESI ions have different internal energies is doubtful, based on studies of internal energy cooling in a QITMS (vide supra). The cited works show with theory and experiment that the time-frame for excited ions to relax to equilibrium internal energy via collisional cooling at $1.33 \times 10^{-3} \mathrm{mbar}$ is about $10 \mathrm{~ms}$. Ongoing research in our laboratory on cooling rates of excited peptide ions indicates that at $9.3 \times 10^{-4}$ mbar equilibrium internal energy is reached in about 20 milliseconds [31]. Therefore, if the ion internal energies imparted by APMALDI and nano-ESI are the same, then any difference in dissociation behavior must be due to different critical energies of dissociation, which may be attributable to different ion conformations. The plots in Figure 2e, $f$, and $h$, shows fragmentation efficiency versus CID delay time (it is being called "CID delay time" rather than "cooling time" since the latter happens in a much shorter time period than the time range shown in the plots). These plots demonstrate little or no change in dissociation as a function of time, suggesting that any excess or difference in internal energy between ionization methods has already been dissipated through collisions with the bath gas. These last data were acquired at the excitation voltage corresponding to $\sim 50 \%$ fragmentation efficiency. One feature of the CID delay time plots is that for most plots the MALDI fragmentation 

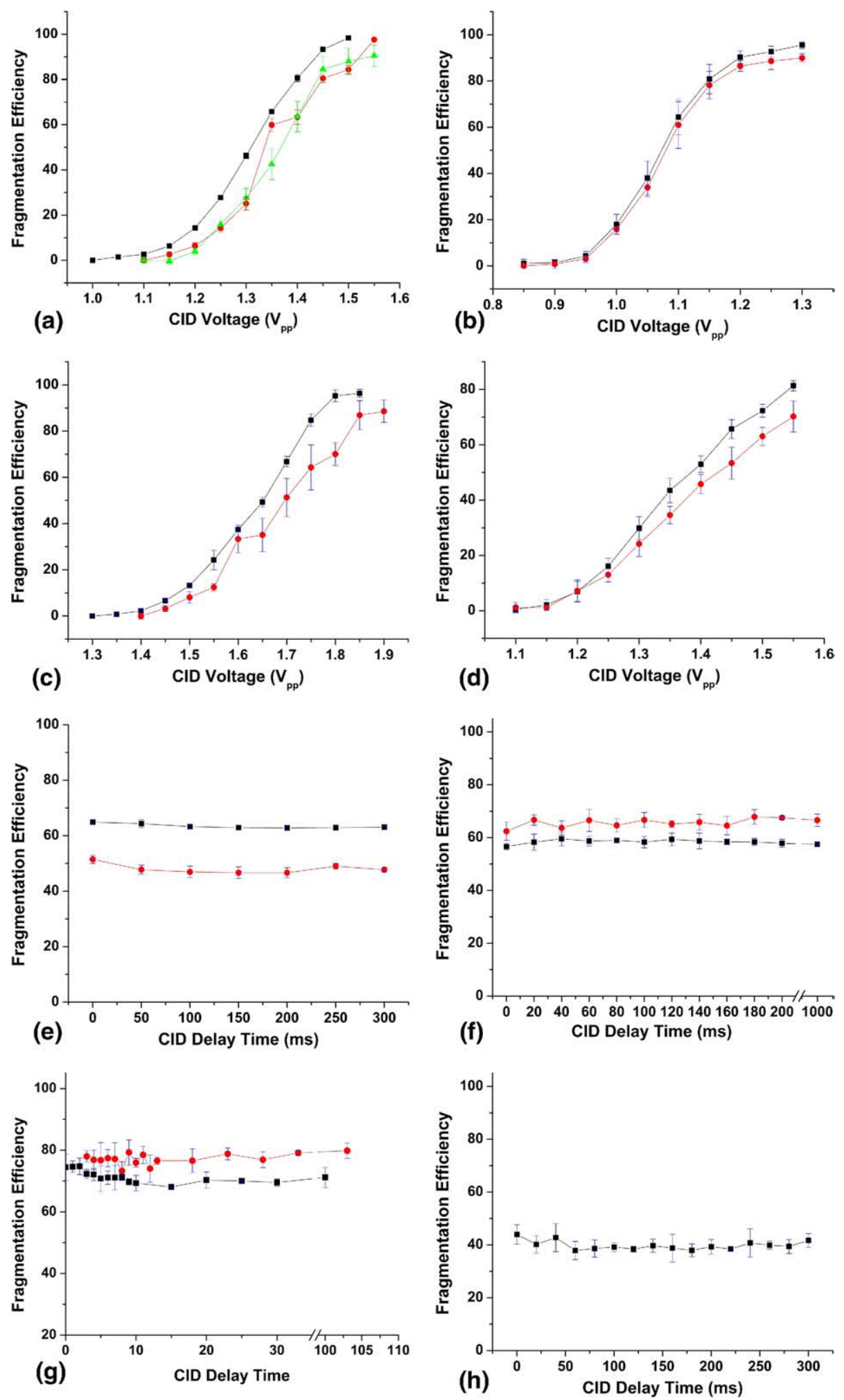

Figure 2. Results of experiments on Bruker Esquire. Filled squares denote nano-ESI, filled circles are AP-MALDI using DHB matrix, and filled triangles are AP-MALDI using CHCA matrix. Plots on the left are of fragmentation efficiency versus CID excitation voltage at $40 \mathrm{~ms}$ excitation time. Plots on the right are of fragmentation efficiency versus CID delay time using the excitation voltage corresponding to $\sim 50 \%$ fragmentation efficiency. Analytes are, (a) desArg ${ }^{9}$ bradykinin, (b) angiotensin III antipeptide, (c) Sar-angiotensin II, (d) desArg ${ }^{1}$ bradykinin, (e) desArg ${ }^{9}$ bradykinin, (f) angiotensin III antipeptide, (g) angiotensin III antipeptide, $1 \mathrm{~ms}$ ion accumulation nano-ESI, $4 \mathrm{~ms}$ ion accumulation AP-MALDI, no parent ion isolation, (h) desArg ${ }^{1}$ bradykinin. 
efficiency is higher, whereas for the fragmentation efficiency versus voltage data the ESI fragmentation is typically higher. This may be due to a shifting in ion flux from the source over time. The MALDI ion populations especially tended to decrease gradually over time and from day-to-day which would shift the optimum excitation frequency for CID. Unfortunately, the use of automated gain control of the ion populations was precluded by the use of MALDI, and obtaining a resonance absorption curve was not an option with the Bruker software. The important conclusion from these data remains that fragmentation was not observed to change with CID delay time before CID, and there was minimal difference in excitation voltage required to fragment ions produced by both ionization techniques.

Figure $2 \mathrm{~g}$ shows fragmentation efficiency versus cool time with shorter ion accumulation times, no parent ion isolation step in the scan function, and $10 \mathrm{~ms}$ CID, to minimize the amount of collisional cooling possible before dissociation. In this case, a slight decrease in dissociation is observed in the first $10 \mathrm{~ms}$, after which fragmentation efficiency stabilizes. Thus, even under the limitation of minimum collisional cooling before dissociation, there are no significant changes in fragmentation efficiency over time.

The present results also contradict recent conclusions made by Konn et al. on the effects of ionization mechanism on internal energy and cool time before CID [14]. Differences in " $50 \%$ fragmentation efficiency" points between nano-ESI and AP-MALDI ions were explained based on differences in gas-phase basicities between the analyte and the MALDI matrix, which has shown some correlation to ion internal energy in a study of dissociation rate of a dinucleotide with several different matrices [32]. However, that work was carried out in an FT-ICR spectrometer at an approximate pressure of $1.3 \times 10^{-8} \mathrm{mbar}$, which did not permit ions to thermalize before CID. Thus, any differences in internal energy deposition resulting from ionization could be preserved. The conditions of AP-MALDI and $\sim 1.3 \times 10^{-3}$ mbar storage in a QITMS are markedly different than conventional MALDI under high vacuum conditions in an FT-ICR spectrometer. Based on the FT-ICR results the observation of Konn and coworkers [14] of dramatic changes in fragmentation efficiency versus cool time over several hundred ms would lead one to conclude that there are differences in internal energy lasting for long periods of time. An initial increase in dissociation was observed in the first $100 \mathrm{~ms}$, followed by a gradual decrease in dissociation extending out to 250 and even $1000 \mathrm{~ms}$ in one example. However, the next series of experiments described will show that ion secular frequency can change substantially under high space charge conditions, which is both interesting in itself and may also offer an explanation for the results of Konn and coworkers [14], which is consistent with the previously determined cool times of ions in a QITMS.
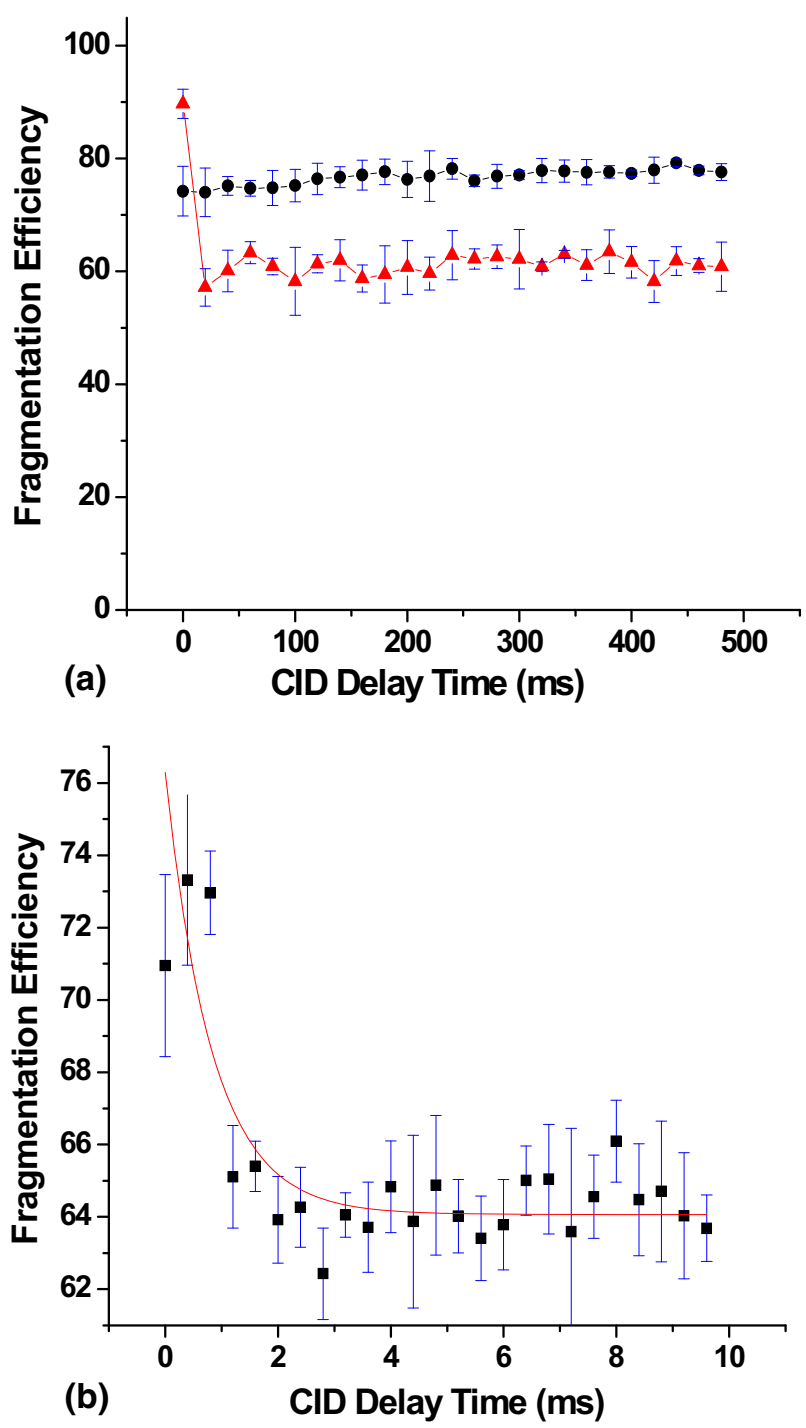

Figure 3. Effect of delay time before CID on fragmentation efficiency. (a) des $\mathrm{Arg}^{1}$ bradykinin, filled circles and filled triangles correspond to $20 \mathrm{~ms}, 590 \mathrm{mV}_{p-p}$ and $200 \mathrm{~ms}, 400 \mathrm{mV}_{p-p}$ ion accumulation time and excitation voltage, respectively; (b) angiotensin III antipeptide, short time scale experiment. Solid red curve is exponential decay fit to data with decay constant of $1197 \mathrm{~s}^{-1}$.

\section{Effect of Ion Population on Cooling Curves}

Figure 3a shows fragmentation efficiency of desArg ${ }^{1}$ bradykinin versus CID delay time for ion accumulation times of 20 and $200 \mathrm{~ms}$, using the Finnigan ITMS. The trace for $200 \mathrm{~ms}$ ion accumulation time shows a dramatic decrease in the first few $\mathrm{ms}$ after ion isolation and then levels off. In Figure 3b, the same initial drop in fragmentation efficiency is observed for the angiotensin III antipeptide with a $200 \mathrm{~ms}$ ion accumulation time. Fragmentation is high for the first three points, followed by a rapid decrease to a base level by $\sim 2 \mathrm{~ms}$. The shape of the plot in Figure $3 \mathrm{~b}$ resembles the results reported by Konn et al. [14] except that the time scales differ by two orders of magnitude. Their interpretation 
was that an initial increase in dissociation after isolation could be caused by the kinetic dampening of the ion cloud after parent isolation at higher $\mathrm{q}_{\mathrm{z}}$ value. While that explanation could be part of the answer, the disparity between the results for large and small ion populations in Figure 3a raises the possibility that space charge effects are playing a significant role. Space charge has been used as a tool for effecting dissociation, via a technique called multipole storage assisted dissociation (MSAD) [33]. MSAD is a method of doing CID primarily in hexapole or octopole ion storage devices, wherein space charge causes the ion cloud to expand, resulting in acceleration of the ions at larger radii where the electric field is larger. Subsequent higher energy collisions with background neutral gas then cause fragmentation [34]. Hexapole and octopole ion traps, due to the inherent nonlinear relationship between radial position and electric field, might be expected to exhibit MSAD more effectively than a quadrupole, where the relationship is linear between electric field and radial position. Nevertheless, MSAD has been observed in a linear quadrupole [35]. Yet, with both the instruments used in the current study, no dissociation was observed when large ion populations were trapped and allowed time to fragment without any supplementary excitation waveform. Consequently, an alternative hypothesis is that ion secular frequencies could be changing over time as the ion cloud expands and condenses, resulting in varying overlap between ion secular frequency and resonant excitation frequency.

\section{Experimentally Measured Shifts in Secular Frequency}

The effect of varying the frequency of the CID excitation voltage for desArg ${ }^{1}$ bradykinin in the Finnigan ITMS is shown in Figure $4 \mathrm{a}$ and $\mathrm{b}$. Both plots display two curves, one representing no CID delay time (squares), and a second showing $100 \mathrm{~ms}$ between isolation and CID (circles). Figure $4 \mathrm{a}$ uses a $50 \mathrm{~ms}$ ion accumulation time, while Figure 4b uses $200 \mathrm{~ms}$ ion accumulation time. Different excitation voltages were required to give comparable fragmentation efficiencies at the no cool time and $100 \mathrm{~ms}$ cool time, but the frequency of maximum fragmentation efficiency did not significantly shift at these excitation voltages. A more thorough analysis would map the fragmentation as a function excitation frequency and voltage at each CID delay time. The reason that less excitation voltage is required at the no cool time condition is because the ions have increased kinetic and internal energy due to the isolation event. In Figure 4a, a shift in the center of the peak of $-150 \mathrm{~Hz}$ is apparent at the longer CID delay time. In Figure $4 \mathrm{~b}$ a more pronounced shift of $-400 \mathrm{~Hz}$ at the longer ion accumulation time is observed. While it is also possible that shifts are due to the increased resonant excitation amplitude [36], this seems unlikely because that should also increase the width of the
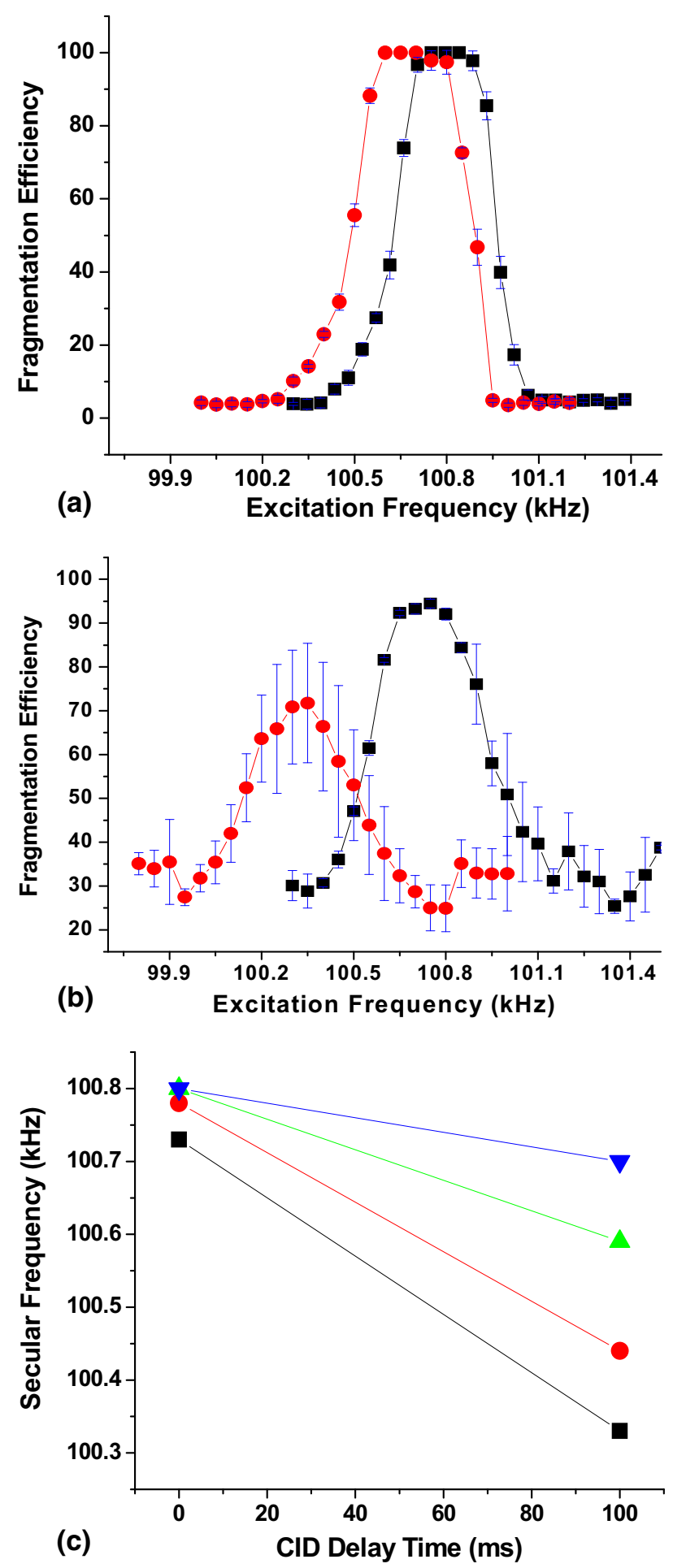

Figure 4. Fragmentation efficiency versus CID resonant excitation frequency, (filled squares) are no delay between isolation and CID, (filled circles) are $100 \mathrm{~ms}$ delay between isolation and CID (a) $50 \mathrm{~ms}$ ion accumulation time, $400 \mathrm{mV}_{p-p}$ (squares), $800 \mathrm{mV}_{p-p}$ circles, (b) $200 \mathrm{~ms}$ ion accumulation time, $400 \mathrm{mV}_{p-p}$ (squares), $1600 \mathrm{mV}_{p-p}$ (circles), (c) peak of dissociation efficiency versus CID delay time for several ion gating times. Squares are $200 \mathrm{~ms}$, circles are $150 \mathrm{~ms}$, up-triangles are $100 \mathrm{~ms}$, and down-triangles are $50 \mathrm{~ms}$.

distribution and make it more asymmetrical, which does not occur here. Figure $4 c$ is a summary of these results for four different ion accumulation times, where 
the frequency corresponding to the center of the peak in fragmentation efficiency is plotted for no cool time and for $100 \mathrm{~ms}$ cool time before CID. The trend is an increasingly larger shift to lower frequencies as ion population increases. These data could be explained if one considered that during the parent isolation event, the parent ions would be axially excited due to offresonant power absorption of the supplementary voltage used to eject the unwanted ions from the trap. This axial expansion would separate the parent ions in space, minimizing their interactions. When the supplementary voltage is removed and the rf voltage is lowered (see Figure 1), the ion cloud would eventually condense down to a smaller volume, where the interactions between the ions are maximized. As the ion cloud goes from a large-volume to small-volume, an increase in dc trapping potential and decrease in secular frequency would be expected.

\section{Ion Trajectory Simulations}

A portion of the scan function from Figure 1 was simulated in SIMION 7.0, using the parameters described in the Methods section. An ion with $m / z 905$ and collision cross section $227 \AA^{2}$ (to simulate desArg ${ }^{1}$ or desArg ${ }^{9}$ bradykinin) was trapped for $3 \mathrm{~ms}$ at $\mathrm{q}_{\mathrm{z}}=0.598$, while a supplementary ac voltage corresponding to $\mathrm{q}_{\mathrm{z}}=0.605(256,989 \mathrm{~Hz})$ and $6.0 \mathrm{~V}_{\mathrm{p}-\mathrm{p}}$ was applied in a dipolar fashion to the endcap electrodes. After $3 \mathrm{~ms}$, the supplementary voltage was turned off and the rf voltage was dropped to $a \mathrm{q}_{\mathrm{z}}=0.253$ for $37 \mathrm{~ms}$. The results for the simulation of a single ion are displayed in Figure 5. Figure 5a shows the evolution of ion kinetic energy, Figure $5 b$ shows axial position, and Figure $5 c$ shows radial position. The ion kinetic energy is highest during the first $3 \mathrm{~ms}$, when off-resonance excitation from the supplementary ac voltage is absorbed and causes acceleration of the ion in a beat-like pattern (the beats are not visible on the scale of this plot). When the supplementary voltage is turned off and the rf voltage dropped to $\mathrm{q}_{\mathrm{z}}=0.253$, the ion kinetic energy begins to subside due to collisions with the He buffer gas, while the ion axial excursions very quickly jump up to higher values before decreasing. The ion radial position is not substantially altered over the course of the entire experiment, which is not surprising given that the excitation from the supplementary voltage was in the axial direction, and ion motion in the axial and radial dimensions is uncoupled in a pure quadrupolar field. The initial expansion of ion motion in the axial direction after dropping the rf voltage is due to the accompanying drop in the depth of the pseudopotential trapping well, given in eq 7 . Figure $5 \mathrm{~d}$ is a histogram of ion axial positions during various parts of the parent isolation experiment. The distribution of medium width is during isolation, the wide distribution is immediately after isolation, and the narrow distribution is after equilibrium has been reached. Although not apparent in Figure 5, when many ions with varied initial positions and veloci-
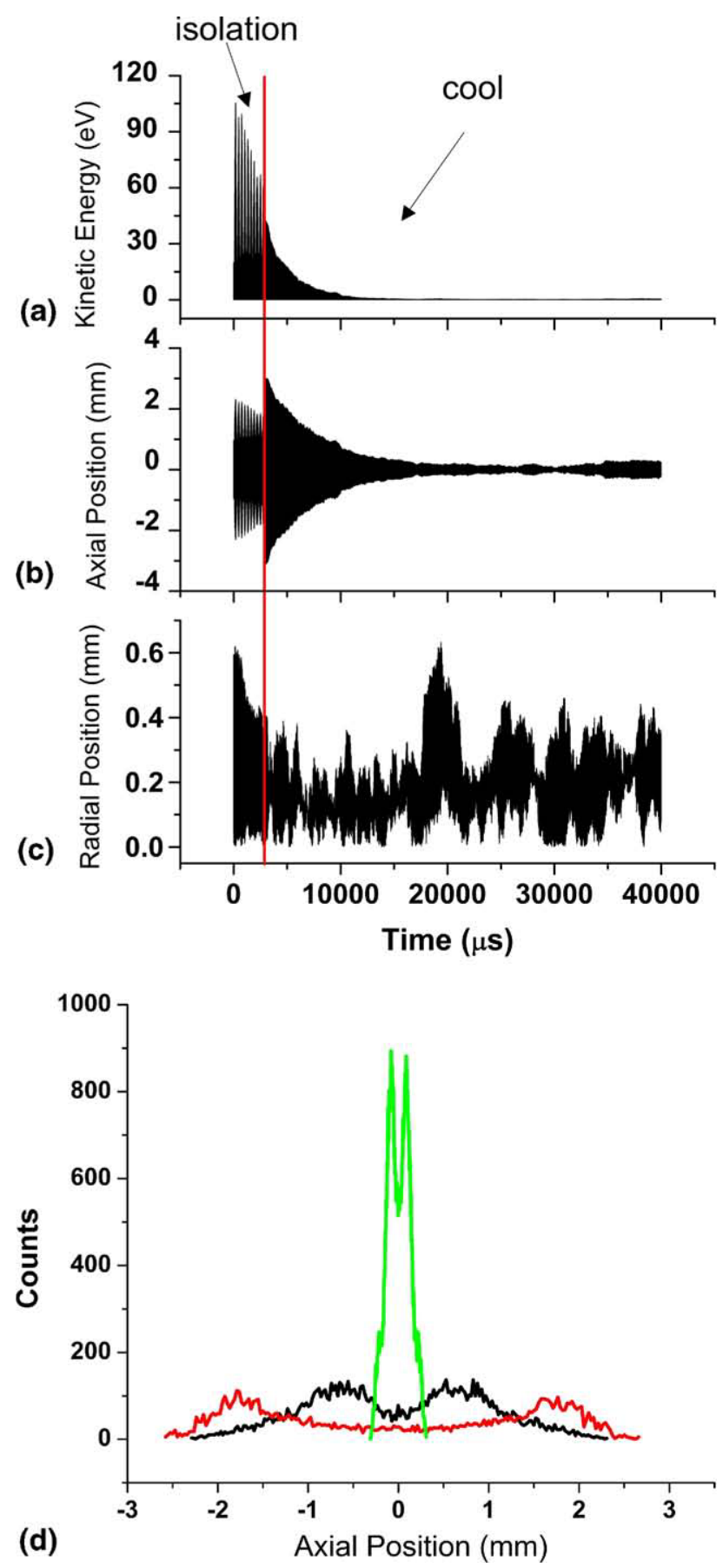

Figure 5. Simulation of parent isolation. Ion parameters given with respect to time. (a) Kinetic energy, (b) axial position, (c) radial position, (d) histogram of ion axial positions during isolation (middle curve), immediately after isolation (wide curve), and at equilibrium (narrow curve).

ties are simulated simultaneously, the dished shape of each excitation and equilibrium distribution blends together into a more Gaussian-shaped curve, and the distribution immediately after isolation extends out to 7 $\mathrm{mm}$. When doing a simulation using $40 \mathrm{~ms}$ of trapping time, computational expense dictates that only one ion is simulated at a time. Regardless of how many ions are 
simulated simultaneously, the salient point from these simulations is that the changing trapping environment used to isolate a parent ion for CID has a noticeable effect on the size of the ion cloud. Immediately after isolation, the high kinetic energy retained from offresonance excitation coupled with the drop in pseudopotential well depth results in an axially diffuse distribution of ions. Through collisions with bath gas helium neutrals, the ions collapse down to the center of the trap, becoming much more concentrated.

\section{Theoretical Shift in Secular Frequency}

The effect of space charge on ion motion was investigated with ion trajectory simulations by using the Coloumbic repulsion model described in the Methods section. Ten ions were simulated at once in a QITMS for $2 \mathrm{~ms}$ at $\mathrm{q}_{\mathrm{z}}=0.253$. The amount of charge, or number of ions represented by each ion, was varied from $1.6 \times$ $10^{-19} \mathrm{C}$ to $1.6 \times 10^{-15} \mathrm{C}$. Therefore, the simulation assumes that the total number of ions in the QITMS was varied from 10 to 100,000 ions. Fourier analysis was performed on the time trace of each ion's axial position, and the average of each frequency domain spectrum was plotted, as shown in Figure 6a. The solid trace is 10 ions, dashes are 10,000 ions, dots are 25,000 ions, dash-dot is 50,000 ions, and dash-dot-dot is 100,000 ions. A broadening of the peak and shift towards lower frequencies is observed as the "number" of ions in the trap is increased. The average frequency values for each condition are plotted in Figure $6 \mathrm{~b}$ (95\% confidence intervals). There is no significant change in frequency until 10,000 ions, after which the average frequency drops linearly as ion number is increased. The greatest experimentally observed shift in ion secular frequency was $400 \mathrm{~Hz}$, shown in Figure 4. Because the exact number of ions in the experiment is not known, one must conclude that either the SIMION simulation is overestimating the effect of space charge, or under space charge conditions the QITMS only holds about 5000-10,000 ions. Estimations of the expected frequency shift versus number of ions are shown in Figure $6 c$ and d, using eqs 11 and 12, respectively. Figure 6c assumes a spherical distribution of constant charge density, and Figure $6 \mathrm{~d}$ uses a Gaussian-shaped axial distribution of a given width $(3 \sigma)$. The largest frequency shift in Figure $6 \mathrm{c}$ is $120 \mathrm{~Hz}$ for 100,000 ions in a sphere of radius 0.40 $\mathrm{mm}$. The voltage induced by larger spheres decreases to the point where almost no frequency shift is calculated for a $6 \mathrm{~mm}$ sphere with 100,000 ions. The frequency shifts in Figure $6 \mathrm{~d}$ are much more pronounced than in Figure $6 c$, because the ions are more concentrated at the center of the Gaussian distributions. A realistically sized distribution [27] with outer radius of $1 \mathrm{~mm}$ gives
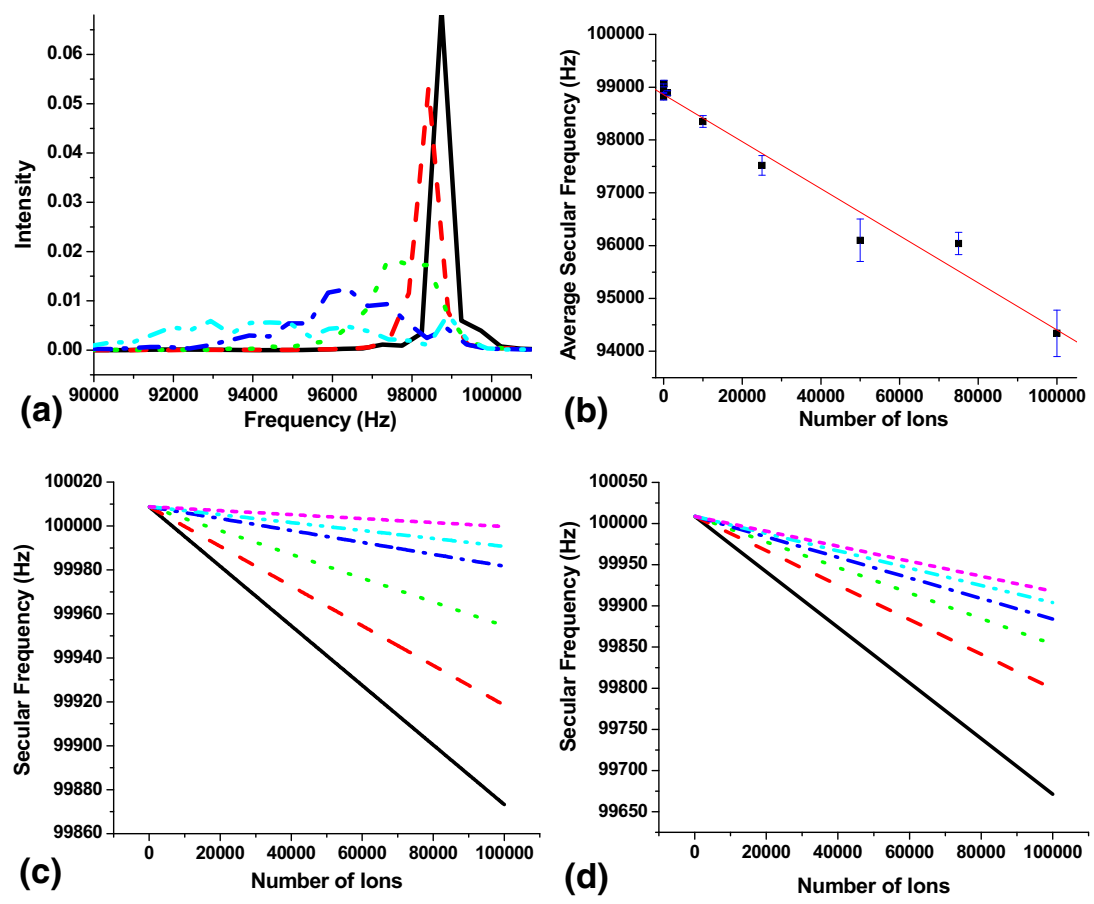

Figure 6. (a) Fourier transform of simulated ion axial positions over a $2 \mathrm{~ms}$ time period. The solid trace is 10 ions, dashes are 10,000 ions, dots are 25,000 ions, dash-dot is 50,000 ions, and dash-dot-dot is 100,000 ions. (b) Average frequency for 10 simulated ion trajectories versus number of ions in trap. (c) Calculation of secular frequency versus number of ions for sphere of constant charge density. Solid trace is $0.4 \mathrm{~mm}$ radius, dash is $0.6 \mathrm{~mm}$, dot is $1.0 \mathrm{~mm}$, dash-dash-dot is $2.0 \mathrm{~mm}$, dash-dot-dot is $3.0 \mathrm{~mm}$, and small dash is $6.0 \mathrm{~mm}$. (d) Calculation of secular frequency versus number of ions for Gaussian distribution of ions. Solid trace is $1.0 \mathrm{~mm}$ outer radius, large dash is $2.0 \mathrm{~mm}$, dot is $3.0 \mathrm{~mm}$, dash-dot is $4.0 \mathrm{~mm}$, dash-dot-dot is $5.0 \mathrm{~mm}$, and small dash is $6.0 \mathrm{~mm}$. 
a shift of $400 \mathrm{~Hz}$ at 100,000 ions. An experiment to estimate the actual number of ions contained in the QITMS under various conditions would be helpful in judging the relative merits of the various theoretical methods employed. However, all the methods concur on the point that a decrease in secular frequency is to be expected as the density of ions increases.

\section{Conclusions}

Many factors contribute to the internal energy of ions in a QITMS. Chief among these factors is the energytransfer process between the ion and the neutral helium bath gas molecules. Evidence has been presented that upon introduction of ions into the QITMS, equilibrium ion internal energy is reached fairly rapidly, about $\sim 10$ ms. Peptide ions produced by nano-ESI and AP-MALDI dissociate with nearly the same CID onset voltage and $50 \%$ fragmentation efficiency point, although in some cases the AP-MALDI ions require more excitation voltage to dissociate. If collisional cooling of internally excited ions occurs in a few ms, as experimental and theoretical studies suggest, then the differences in dissociation of ions produced by nano-ESI and AP-MALDI must be due to different ion conformations [30].

Typical QITMS operations such as parent ion isolation for CID may increase the kinetic energy of the ions, possibly increasing the ion internal energy as well. However, because the rate of internal energy dissipation appears to be fast, only slight variations in dissociation efficiency are observed when a cool time is inserted between isolation and CID.

Injecting large numbers of ions into a QITMS increases the space charge in the trap, which can add an effective dc offset to the trapping voltage, altering the secular frequencies of ion oscillation. Because a voltage induced by a cloud of ions depends on the ion density, parameters which affect the size of the ion cloud will change the induced dc offset. When ion populations were very large, decreases in secular frequency of several hundred Hertz were observed, by measuring immediately after ion isolation and at $100 \mathrm{~ms}$ after ion isolation. This change in secular frequency could make estimations of relative ion internal energy problematic, particularly if the method used depends on a resonance condition being achieved between the frequency of excitation voltage and ion secular frequency. We believe the conclusions of Konn et al. [14] are incorrect because they had too many ions in their experiments and their purported changes in internal energy were in fact artifacts of the measurement. The changes they measured were a result of shifts in secular frequency, not changes in internal energy.

\section{Acknowledgments}

The authors acknowledge support for this work by NSF grant no. CHE-0431825.

\section{References}

1. McLafferty, F. W.; Wachs, T.; Lifshitz, C.; Innorta, G.; Irving, P. Substituent Effects in Unimolecular Ion Decompositions. XV. Mechanistic Interpretations and the Quasi-Equilibrium Theory. J. Am Chem. Soc. 1970, 92, 6867-6880.

2. Baer, T.; Mayer, P. M. Statistical Rice-Ramsperger-Kassel-Marcus QuasiEquilibrium Theory Calculations in Mass Spectrometry. J. Am. Soc. Mass Spectrom. 1997, 8, 103-115.

3. Baer, T.; Hase, W. L. Unimolecular Reaction Dynamics; Oxford University Press: New York, 1996.

4. Marcus, R. A.; Rice, O. K. Session on Free Radicals, The Kinetics of the Recombination of Methyl Radical and Iodine Atoms. J. Phys. Colloid Chem. 1951, 55, 894-908.

5. Laskin, J.; Futrell, J. Internal Energy Distributions Resulting from Sustained Off-Resonance Excitation in Fourier Transform Ion Cyclotron Resonance Mass Spectrometry. II. Fragmentation of the 1-Bromonaphthalene Radical Cation. J. Phy. Chem. A 2000, 104, 5484-5494.

6. Laskin, J.; Byrd, M.; Futrell, J. Internal Energy Distributions Resulting from Sustained Off-Resonance Excitation in FTMS. I. Fragmentation of the Bromobenzene Radical Cation. Int. J. Mass Spectrom. 2000, 195/196, 285-302.

7. Vestal, M. L. Theoretical Studies on the Unimolecular Reactions of Poly-Atomic Molecule Ions. J. Chem. Phys. 1965, 43, 1356-69.

8. Plass, W. R.; Cooks, R. G. A Model for Energy Transfer in Inelastic Molecular Collisions Applicable at Steady State or Non-Steady State and for an Arbitrary Distribution of Collision Energies. J. Am. Soc. Mass Spectrom. 2003, 14, 1348-1359.

9. Stafford, G. C. J.; Kelley, P. E.; Syka, J. E. P.; Reynolds, W. E.; Todd, J. F. J. Recent Improvements in and Analytical Applications of Advanced Ion Trap Technology. Int. J. Mass Spectrom. Ion Processes 1984, 60, 85-98.

10. March, R. E. An Introduction to Quadrupole Ion Trap Mass Spectrometry. J. Mass Spectrom. 1997, 32, 351-369.

11. Goeringer, D. E.; McLuckey, S. A. Relaxation of Internally Excited High-Mass Ions Simulated Under Typical Quadrupole Ion Trap Storage Conditions. Int. J. Mass Spectrom. 1998, 177, 163-174.

12. Black, D. M.; Payne, A. H.; Glish, G. L. Determination of Cooling Rates in a Quadrupole Ion Trap. J. Am. Soc. Mass Spectrom. 2006, 17, 932-938.

13. Remes, P. M.; Glish, G. L. Collisional Cooling Rates in a Quadrupole Ion Trap at Sub-Ambient Temperatures. Int. J. Mass Spectrom. 2007, 265, $176-181$.

14. Konn, D. O.; Murrell, J.; Despeyroux, D.; Gaskell, S. J. Comparison of the Effects of Ionization Mechanism, Analyte Concentration, and Ion "CoolTimes" on the Internal Energies of Peptide Ions Produced by Electrospray and Atmospheric Pressure Matrix-Assisted Laser Desorption Ionization. J. Am. Soc. Mass Spectrom. 2005, 16, 743-751.

15. Kato, S.; Bierbaum, V. M.; Leone, S. R. Laser Fluorescence and Mass Spectrometric Measurements of Vibrational Relaxation of $\mathrm{N}_{2}^{+}(\mathrm{V})$ with $\mathrm{He}, \mathrm{Ne}, \mathrm{Ar}, \mathrm{Kr}$, and $\mathrm{Xe}^{*}$. Int. J. Mass Spectrom. Ion Processes 1995, 149/150, 469-486.

16. Stein, S. E.; Rabinovitch, B. S. Accurate Evaluation of Internal Energy Level Sums and Densities Including Anharmonic Oscillators and Hindered Rotors. J. Chem. Phys. 1973, 58, 2438-2445.

17. March, R. E.; Todd, J. F. J. Practical Aspects of Ion Trap Mass Spectrometry; CRC Press: New York, 1997.

18. Major, F. G.; Dehmelt, H. G. Exchange-Collision Technique for the rf Spectroscopy of Stored Ions. Phys. Rev. 1968, 170, 91-107.

19. VanBerkel, G. J.; Glish, G. L.; McLuckey, S. A. Electrospray Ionization Combined with Ion Trap Mass Spectrometry. Anal. Chem. 1990, 62, 1284 1295.

20. Bushey, J. M.; Kaplan, D. A.; Danell, R. M.; Glish, G. L. Pulsed Nano-Electrospray Ionization, Characterization of Temporal Response, and Implementation with a Flared Inlet Capillary. Instrum. Sci. Technol. 2009, 37, 257-273

21. Glish, G. L.; Danell, R. M. Electrospray Ionization Device; U.S. Patent No. 2004, 6,703,611.

22. Danell, R. M. Advances in Ion Source and Quadrupole Ion Trap Performance and Design; Ph.D. Dissertation, The University of North Carolina, 2001.

23. Danell, R. M. An Atmospheric Pressure MALDI Probe for Use with ESI Source Interfaces. Proceedings of the 48th ASMS Conference on Mass Spectrometry and Allied Topics; Long Beach, CA, June 2000.

24. Dahl, D. A. SIMION 3d 7.0, 1999.

25. Manura, D. Collision Model Hs1. http//www.simion.com/info/Collision Model_HS1. 2007.

26. Griffiths, D. J. Introduction to Electrodynamics; Prentice-Hall Inc: Upper Saddle River, 1999.

27. Cleven C. D.; Cooks, R. G.; Garrett, A. W.; Nogar, N. S.; Hemberger, P. H. Radial Distributions and Ejection Times of Molecular Ions in an Ion Trap Mass Spectrometer. A Laser Tomography Study of Effects of Ion Density and Molecular Type. J. Phys. Chem. 1996, 100, 40-46.

28. Li, G.-Z.; Guan, S.; Marshall, A. G. Comparison of Equilibrium Ion Density Distribution and Trapping Force in Penning, Paul, and Combined Ion Traps. J. Am. Soc. Mass Spectrom. 1998, 9, 473-481.

29. Remes, P. M.; Glish, G. L. Mapping the Distribution of Ion Positions as a Function of Quadrupole Ion Trap Mass Spectrometer Operating Parameters to Optimize Infrared Multiphoton Dissociation. J. Phys. Chem. A 2009, 113, 3447-3454. 
30. Danell, A. S.; Glish, G. L. Evidence for Ionization-Related Conformational Differences of Peptide Ions in a Quadrupole Ion Trap. J. Am. Soc. Mass Spectrom. 2001, 12, 1331-1338.

31. Demireva, M. P.; Remes, P. M.; Glish, G. L. Internal Energy Relaxation of Different Mass Peptide Ions in Quadrupole Ion Trap Mass Spectrometry. Proceedings of the 55th ASMS Conference on Mass Spectrometry and Allied Topics; Indianapolis, IN, June 2007.

32. Stevenson, E.; Breuker, K.; Zenobi, R. Internal Energies of Analyte Ions Generated from Different Matrix-Assisted Laser Desorption/Ionization Matrices. J. Mass Spectrom. 2000, 35, 1035-1041.

33. Sannes-Lowery, K.; Griffey, R. H.; Kruppa, G. H.; Speir, J. P.; Hofstadler S. A. Multipole Storage Assisted Dissociation, a Novel in-Source Disso- ciation Technique for Electrospray Ionization Generated Ions. Rapid Commun. Mass Spectrom. 1998, 12, 1957-1961.

34. Håkansson, K.; Axelsson, J.; Palmblad, M.; Håkansson, P. Mechanistic Studies of Multipole Storage Assisted Dissociation. J. Am. Soc. Mass Spectrom. 2000, 11, 210-217.

35. Black, D. Novel Methods for Characterization of Intact Proteins by Mass Spectrometry; Ph.D. Dissertation, The University of North Carolina, 2005.

36. Liere, P.; Blasco, T.; March, R. E.; Tabet, J.-C. Influence of Cooling Time on the Internal Energy of Ions Subjected to Resonance Excitation in a Quadrupole Ion Trap. Rapid Commun. Mass Spectrom. 1994 $8,953-956$. 